\title{
New High Availability Four Quadrant Converter [600A; 10V] for LHC
}

Vicente Raúl Herrero

CERN, Geneva, Switzerland,

Keywords: «Particle accelerator», «Parallel operation», « Converter control», « Power supply».

\begin{abstract}
High availability in four quadrant (4-quadrant) converters is crucial in Large Hadron Collider (LHC) operation. The level of radiation will increase, and higher availability is required for each system. To get this aim, a new redundant 4-quadrant linear converter is developed modifying the control strategy of current converters in LHC. Applied characteristics and experimental results are exposed in this paper.
\end{abstract}

Presented at: EPE 2015, 7-10 September 2015, Geneva, Switzerland

Geneva, Switzerland

October, 2015 


\title{
New High Availability Four Quadrant Converter [600A; 10V] for LHC
}

\author{
Vicente Raúl Herrero \\ CERN \\ Route de Meyrin 385 \\ Meyrin, Switzerland \\ E-Mail: vherrero@cern.ch \\ URL: http://www.cern.ch
}

\section{Acknowledgements}

The author wish to thank CERN TE-EPC group members for the helpful discussions and valuable comments, and their general contribution to this job.

\section{Keywords}

«Particle accelerator», «Parallel operation», « Converter control», « Power supply».

\begin{abstract}
High availability in four quadrant (4-quadrant) converters is crucial in Large Hadron Collider (LHC) operation. The level of radiation will increase, and higher availability is required for each system. To get this aim, a new redundant 4-quadrant linear converter is developed modifying the control strategy of current converters in LHC. Applied characteristics and experimental results are exposed in this paper.
\end{abstract}

\section{Introduction}

CERN will operate the LHC machine after a program of consolidation called "Long Shut Down 1" (LS1), using the collision energy of the particles contained in the accelerator at its initial nominal level of $7 \mathrm{TeV}$ each. Operating conditions will lead to higher levels of radiation over some specific converters. In order to keep constant the machine performance, 4-quadrant redundant converters, exposed to radiations, are required to feed magnetic loads with high availability and current precision. Current precision is in the range of $10 \mathrm{ppm}$ up to $50 \mathrm{ppm}$ (ppm: part per million). A radiant tolerant design is implemented, but this part is out of the scope of this paper.

Existing converters are developed by mean of a 4-quadrant linear topology at the output stage [1], [3], [4], [5]. This topology is kept due to its radiation tolerance performance in opposition the switched topologies. This stage makes use of power mosfet transistors controlled as variable impedances by mean of a voltage regulation loop. The control strategy provides a voltage source that cannot be parallelized. In Fig. 1 the control strategy diagram is presented.

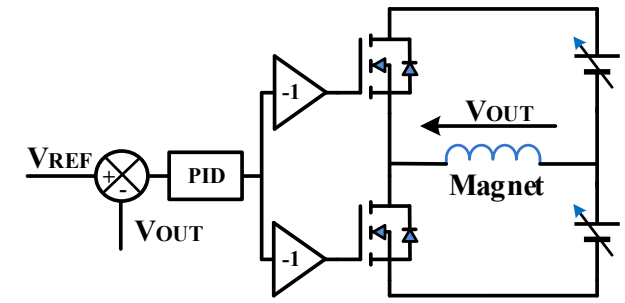

Fig. 1: Control strategy of current 4-quadrant linear converter. Non-parallelizable solution.

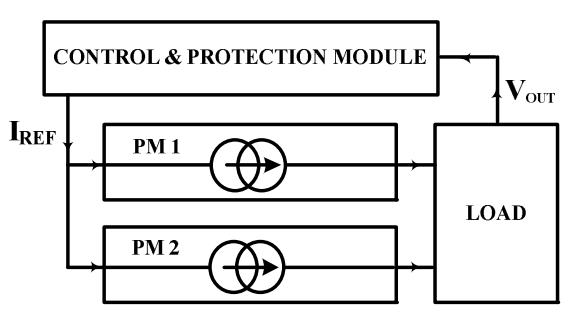

Fig. 2: New 4-quadrant converter structure.

To allow redundant operation, a new converter (R2E-LHC600A-10V) is carried out. Three modules form the new converter structure (Fig. 2): Two power modules which work as parallelizable current sources of $\pm 400 \mathrm{~A}$ 
$\pm 10 \mathrm{~V}$, and one module which controls the output voltage providing the current references to both power modules. The protection elements, that were located in the rack in current converters [1], are placed in the control and protection module. This new structure provides full modularity and reduces the time to repair.

Three stages make up each power module: Input stage, double ended DC/DC stage and 4-quadrant linear stage (Fig. 3).

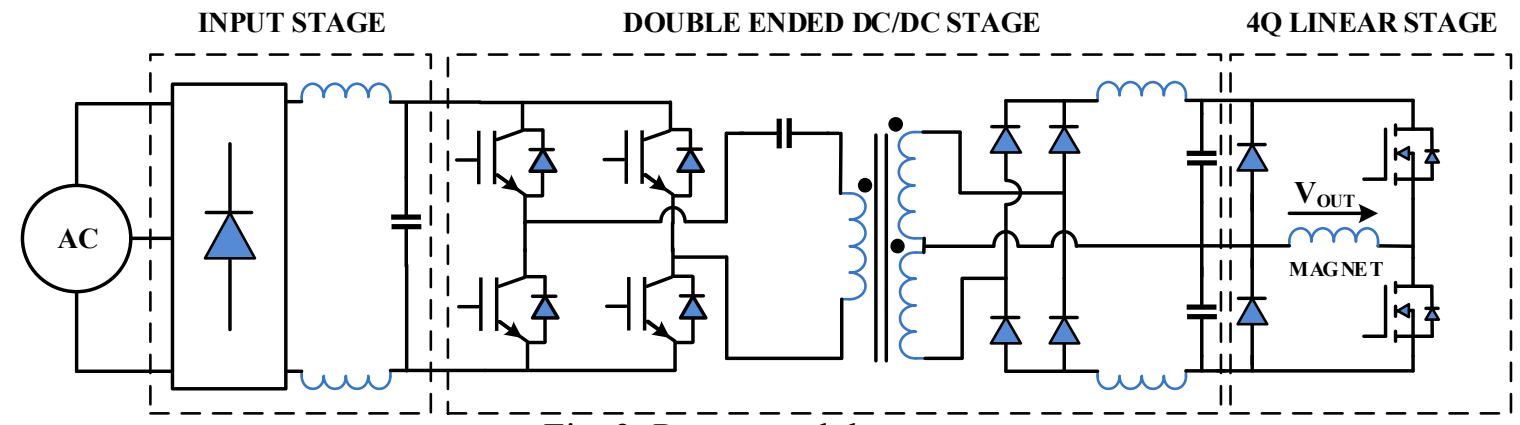

Fig. 3: Power module stages.

In next sections, the principal improvements to comply with the LHC requirements are explained.

\section{New 4-quadrant linear stage topology.}

\section{Electrical structure of the new 4-quadrant linear stage}

A double ended DC/DC followed by two power mosfets working in the active region provide power to the magnetic load. The load has a strongly inductive performance, so it is considered as a current source in the analysis presented in this paper. The mosfets will work with a permanent $\mathrm{V}_{\mathrm{DS}}$ voltage of $2 \mathrm{~V}$ (active region). In this working point the current provided by the mosfet is independent of the $\mathrm{V}_{\mathrm{DS}}$ value (Fig. 4), so it will act as a current source controlled by the gate voltage. Technical rules of electricity forbid feeding a current source (magnet) from other current source (mosfet). To decouple both current sources a capacitor is placed in parallel with the load (magnet). In this way, the current sources that come from the use of the power mosfets, feed a voltage source symbolized by the decoupling capacitor. The voltage source then feeds the current source represented by the inductive load (magnet). A resistor is placed in parallel with the load to damp the resonance created in system. Fig. 5 shows the schema which symbolizes the electrical structure.

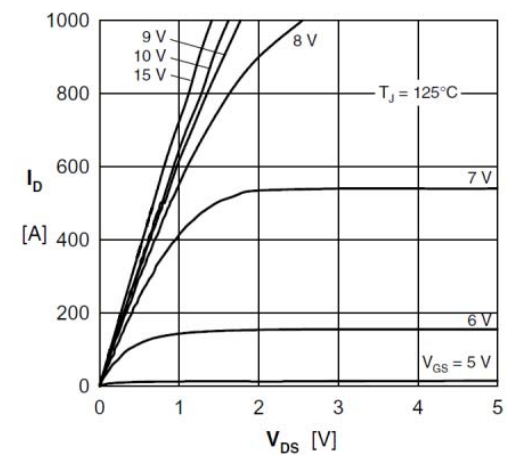

Fig. 4: Drain current VS drain to source voltage in mosfet: VMO1200-01F.

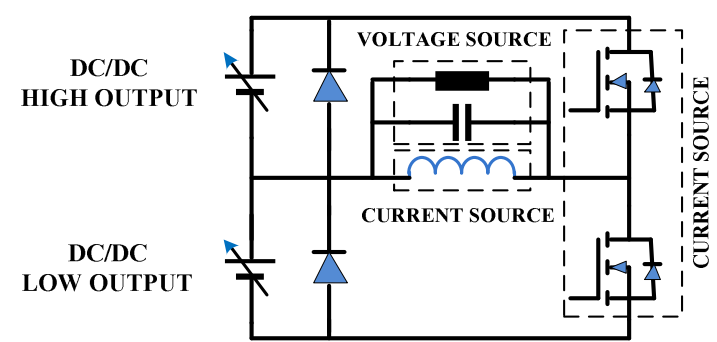

Fig. 5: Electric structure of 4-quadrant linear stage.

\section{Operational principle}

A 4-quadrant converter can work in two different ways, as a generator when it supplies energy to the load and as a receptor when it absorbs the load energy. 


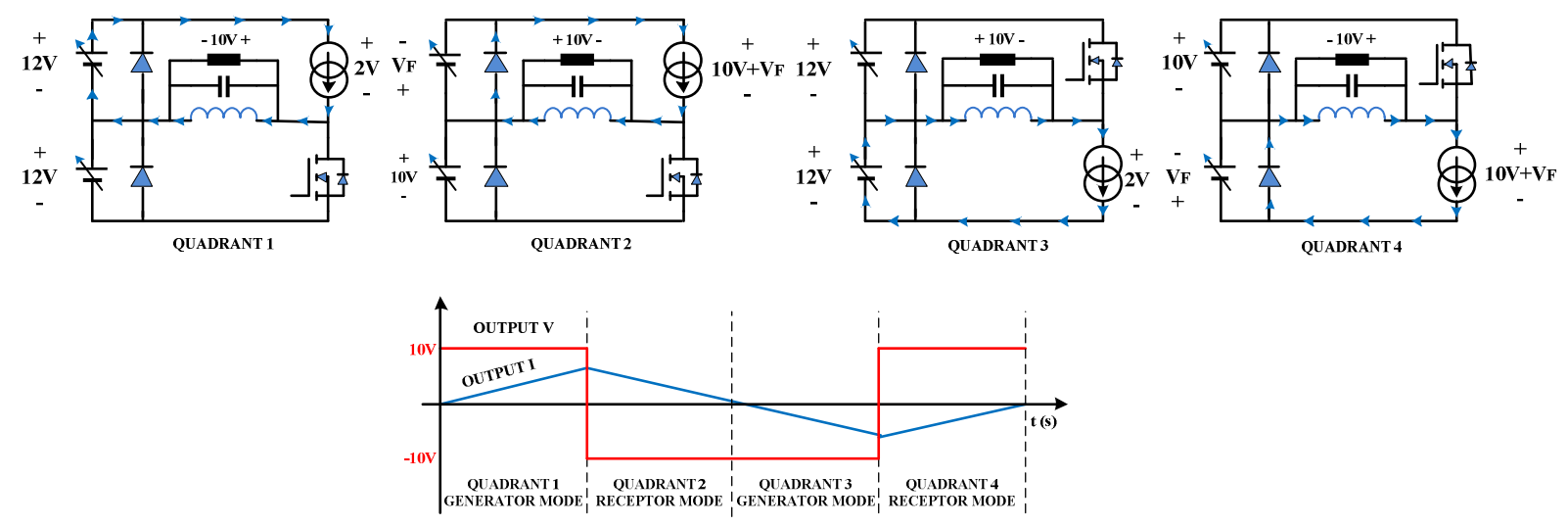

Fig. 6: Equivalent circuit of each quadrant of operation, and example of operative current and voltage in the magnet.

When the converter is working in quadrant 1 or 3 (generator mode) the DC/DC stage is giving power to feed the load. In quadrant 2 or 4 (receptor mode) the DC/DC stage does not provide power, the output current circulates through the free-wheeling diodes that have been added, updating the current topology. In these quadrants the mosfet, symbolized as a current source, controls the voltage applied to the load using the magnet current, and at the same time dissipates the load energy.

\section{Control strategy}

Two overlapped and decoupled loops form the implemented control strategy [2]. The internal loops are located in the power modules and they regulate the current provided by the mosfets. The external loop is inside the control and protection module and regulates the output voltage providing the current references to both power modules. Its dynamical behavior is ten times slower than the internal loops, to keep a stable operation. Fig. 7 shows the control strategy diagram.

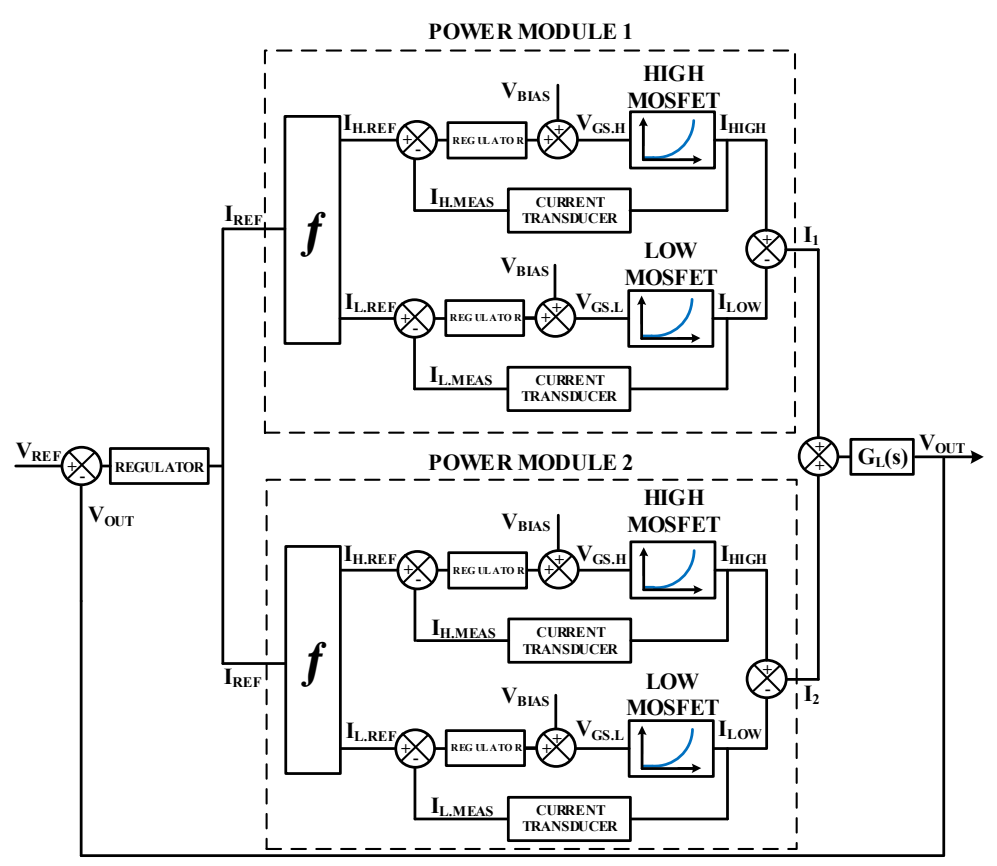

Fig. 7: New Control Strategy for the 4-quadrant 600A, 10V Converter.

\section{Quick reaction in case of power module fail}

In redundant operation, if one power module stops in a failure situation, the operational power module has to supply the full power. The bandwidth of the voltage control loop limits the time to response to this perturbation (Fig. 7). During this time, the current that was controlled by the stopped power 
module is diverted to the decoupling capacitor. By consequence, during this time, the decoupling capacitor is the only element that limits the decreasing of the output voltage. (Fig. 8.b)

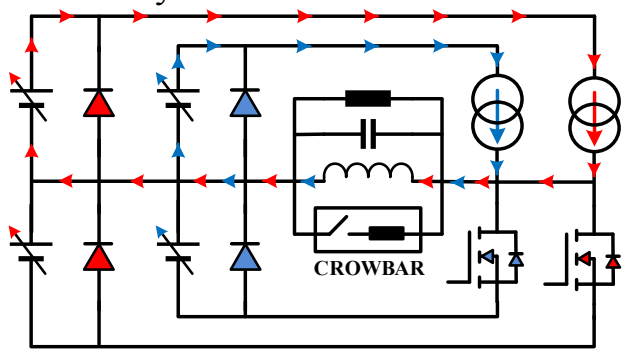

(a)

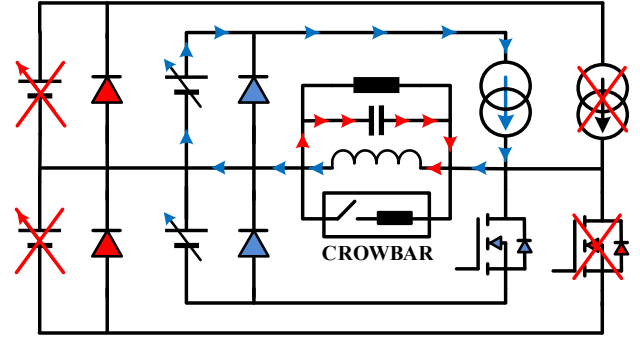

(b)

Fig. 8: Normal operative situation (a) and power module failure operative situation (b).

In the presented converter, a safety crowbar circuit is used to prevent an overvoltage condition in the magnetic load. The system short-circuits the magnetic load by mean of a resistor if the output voltage reaches a threshold value. To warrant that this system is not activated and the converter is not stopped, the output voltage must be kept under this limit value.

If we keep the control strategy shown in Fig. 7 the size of the capacitor needed would be too big. To reduce the decoupling capacitor size, each power module includes a high speed response system to minimize the time during which current is circulating through the decoupling capacitor (Fig. 9). The system operates in the following way: The failure status signal turns on when the opposite module fails. At this moment, the current reference is doubled and the voltage applied to the mosfet gate increases quickly, up to the moment in which the current is double respect to the initial moment of the fail or the mosfet is saturated. After this moment, the regulation elements (Fig. 7) take the control again.

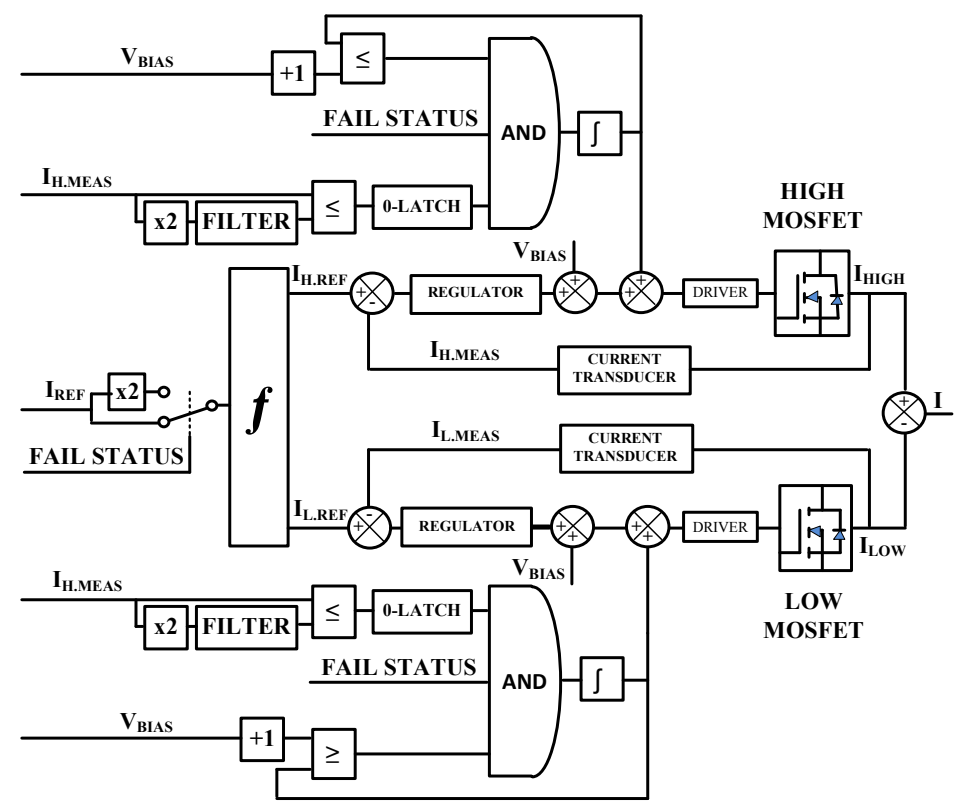

Fig. 9: High speed response system.

\section{Quick reaction in quadrants 1 and 3, generator mode.}

Below the behavior of the high speed response system in the quadrant 1 is explained. 


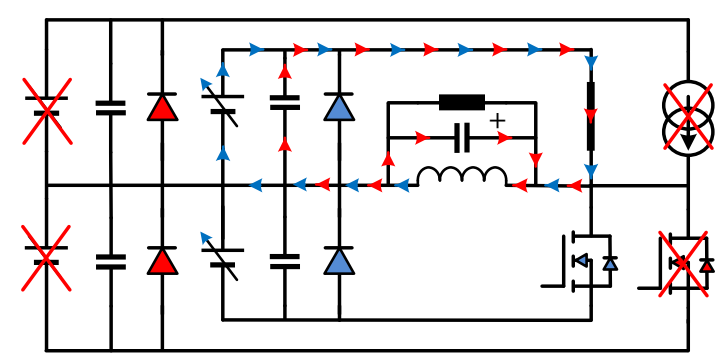

(a) $t_{1} \rightarrow t_{2}$

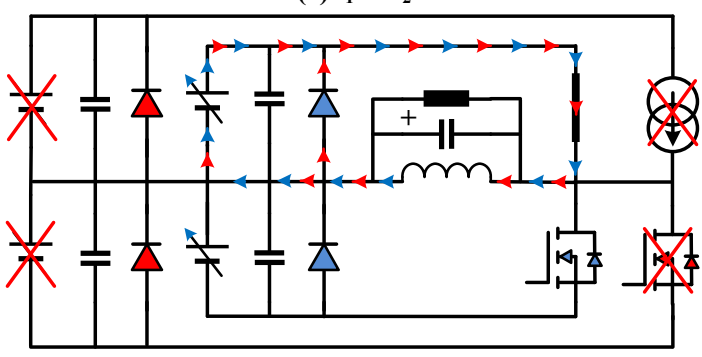

(b) $t_{2} \rightarrow t_{3}$

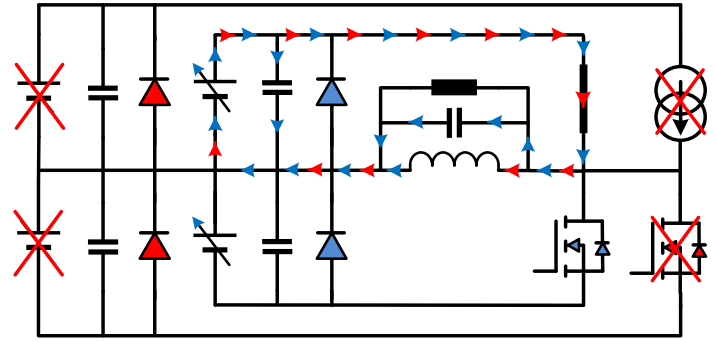

(c) $\mathbf{t}_{3} \rightarrow \mathbf{t}_{4}$

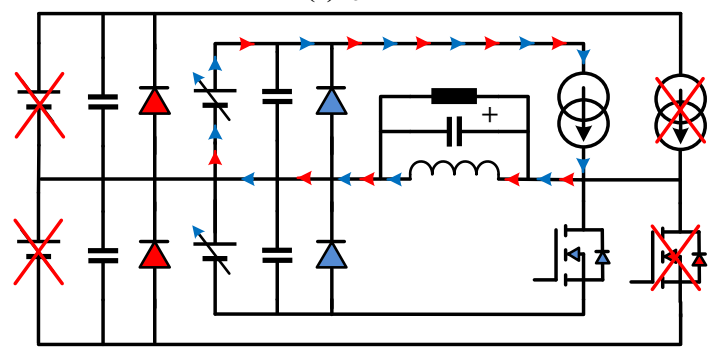

(d) $t_{4} \rightarrow t_{5}$

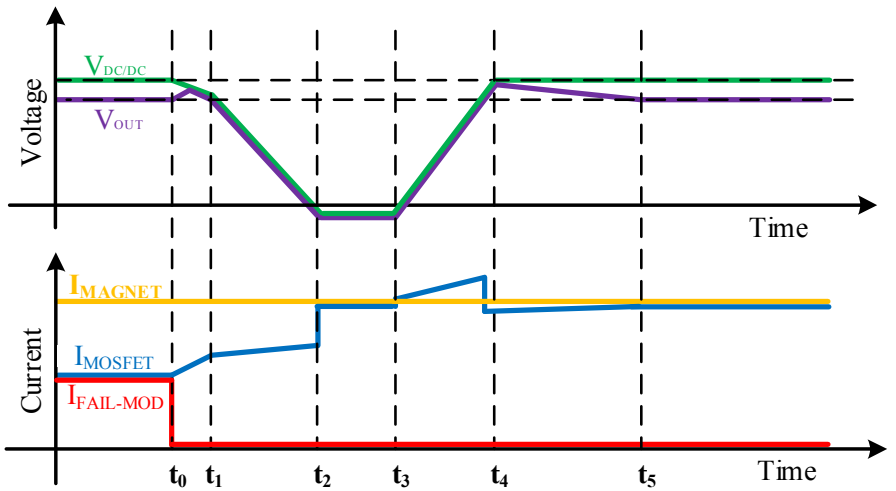

(e)

Fig. 10: Power module fault operational principle in first quadrant.

$\mathrm{t}_{0} \rightarrow \mathrm{t}_{1}$

The current which was circulating by the failed module, is deviated to the decoupling capacitor. In the same moment, the voltage in the mosfet gate is increased saturating the mosfet that starts to act as a short circuit. Therefore the voltages in the DC/DC output capacitor and the decoupling capacitor are the same, if the voltage that falls in the mosfet is denied.

$\mathrm{t}_{1} \rightarrow \mathrm{t}_{2}$

The response of the DC/DC stage is not enough fast to react on time versus a big variation of load. So the extra amount of current that the magnet is providing respect to the one that the $\mathrm{DC} / \mathrm{DC}$ is giving is diverted to the parallelization of both capacitors. This current will make decrease the voltage of both capacitors at the same time.

$\mathrm{t}_{2} \rightarrow \mathrm{t}_{3}$

The voltage in the $\mathrm{DC} / \mathrm{DC}$ output capacitor reaches the forward voltage of the diode which is in parallel with it. In consequence the diode starts to conduct, keeping constant the voltage through the capacitors and causing that all the magnet current crosses the mosfet that continues saturated.

$\mathrm{t}_{3} \rightarrow \mathrm{t}_{4}$

The current provided by the inverter continues increasing and reaches the value of the current in the magnet. After this moment the extra amount of current starts to charge both capacitors up to the moment in which the voltage at the DC/DC stage output is regulated. In this moment the $\mathrm{DC} / \mathrm{DC}$ stage current returns to the stable value giving the same current than the magnet. 
$\mathrm{t}_{4} \rightarrow \mathrm{t}_{5}$

The mosfet continues saturated up to the moment in which the output voltage reaches the reference value. In this moment the 4-quadrant controller starts to decrease the gate voltage in order to control again the magnet voltage. In this moment the system is stable again and the magnet voltage is regulated by mean of only one of the two power modules.

Quick reaction in quadrants 2 and 4, receptor mode.

Below the behavior of the high speed response system in the quadrant 2 is explained.

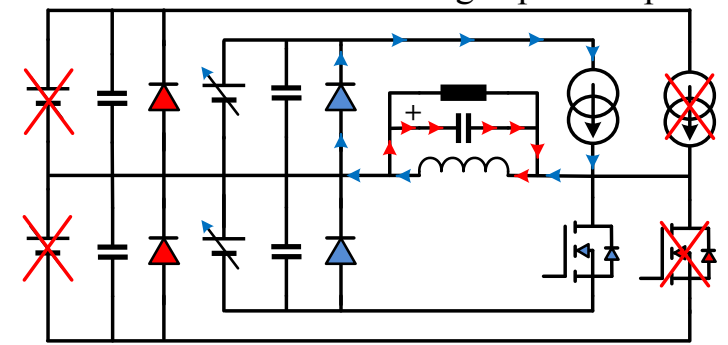

(a) $\mathbf{t}_{0}$

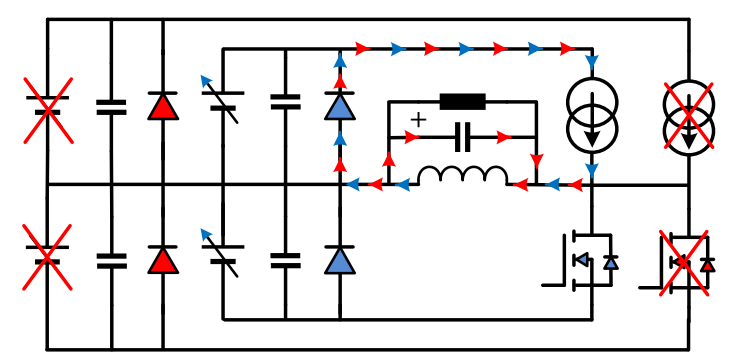

(b) $\mathbf{t}_{0} \rightarrow \mathbf{t}_{1}$

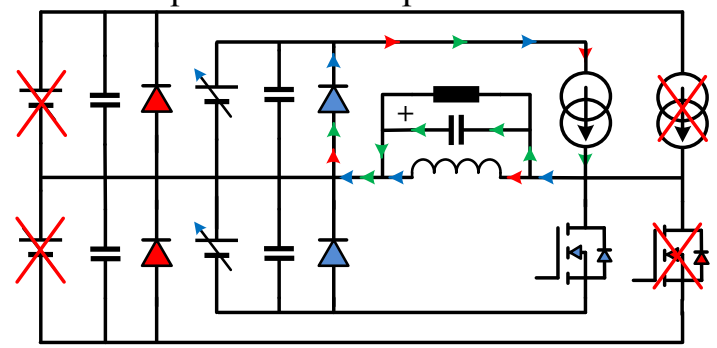

(c) $\mathbf{t}_{1} \rightarrow \mathbf{t}_{2}$

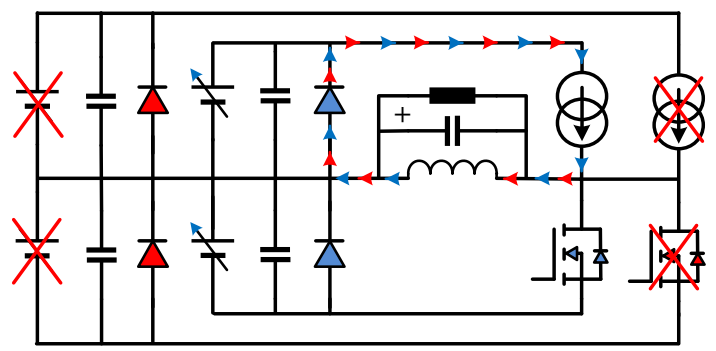

(d) $\mathbf{t}_{3} \rightarrow \mathbf{t}_{4}$

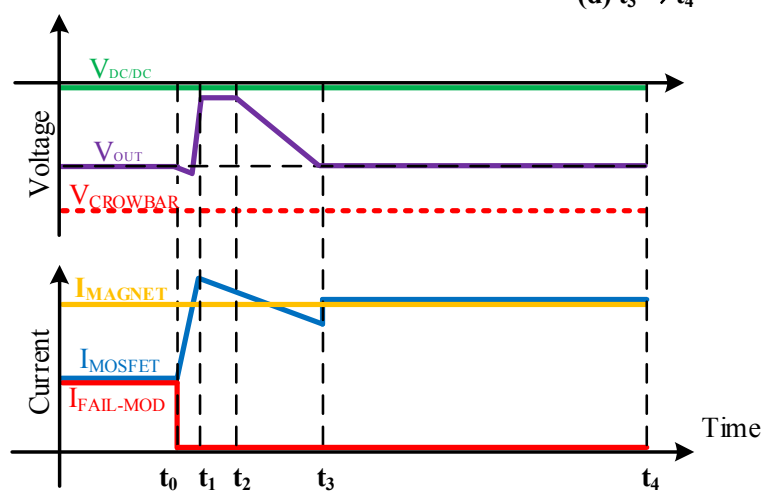

(e)

Fig. 11: Power module fault operational principle in second quadrant.

$\mathrm{t}_{0}$

Working in quadrant 2, the DC/DC stage is deactivated to reduce the amount of energy dissipated in the mosfet; by consequence the current circulates through the output diodes (Fig. 6). When the failure situation arises, the current which circulates in the non-operative module, is deviated to the decoupling capacitor.

$\mathrm{t}_{0} \rightarrow \mathrm{t}_{1}$

The signal of "opposite module in fault" arrives to the operative power module and the high speed response system increase the current in the mosfet. By consequence the decoupling capacitor is discharged.

$\mathrm{t}_{1} \rightarrow \mathrm{t}_{2}$

The high speed response system stops to work, letting the regulation system to control again the magnet voltage. Because the magnet voltage is over the reference, the regulation makes 
decrease the current in the mosfet up to the moment in which the magnet voltage reach the reference value.

$\mathrm{t}_{2} \rightarrow \mathrm{t}_{3}$

The output voltage reaches the reference, and the 4-quadrant stage controls the current by the mosfet in order to be the same than the current that is circulating in the magnet. In this moment the system is stable again and the magnet voltage is regulated by mean of only one of the two power modules.

\section{Experimental results.}

A prototype of the full converter was built to be tested in the laboratory. Fig. 12 shows the arrangement of both power modules in parallel.

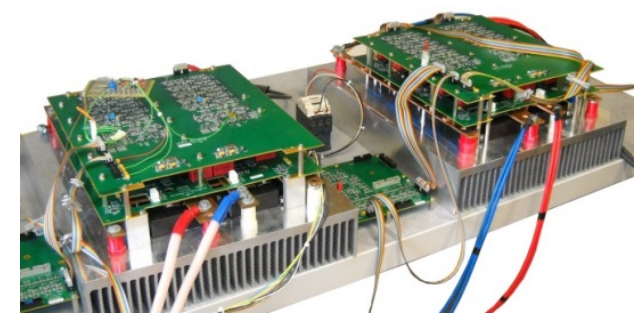

Fig. 12: Prototype of two power modules working in parallel.

\section{Fault in quadrant 1, generator mode.}

The graphs below show the converter behavior when one of the two power modules fails in the first quadrant in two different situations: Before to implement the high speed response system and after the implementation. In the moment of causing the power module fault the working point is $6.2 \mathrm{~V}$ and $400 \mathrm{~A}$ (200A x 2).
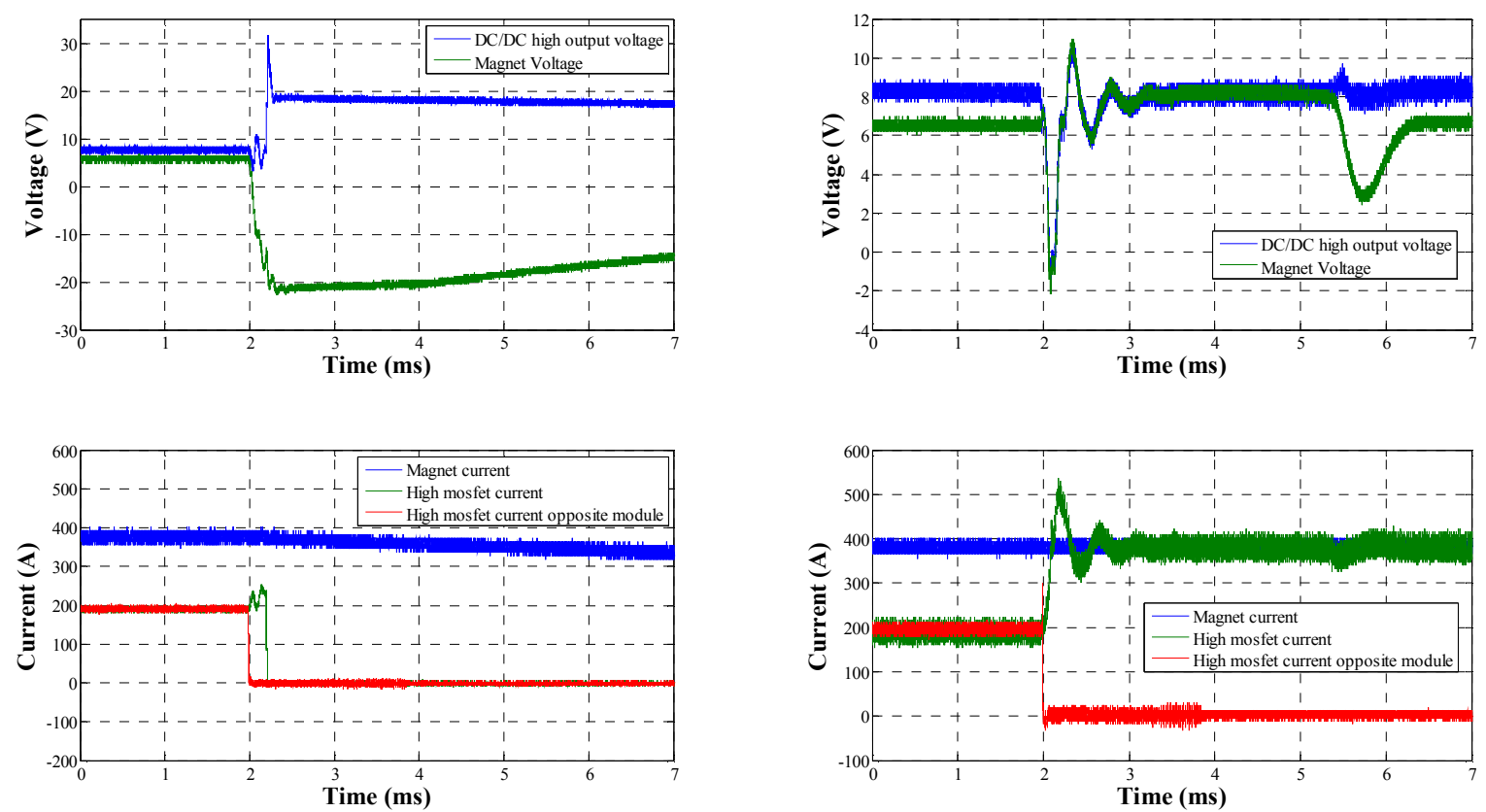

Fig. 13: Comparative of power module fault before (left column) and after (right column) implementing the high speed response system in quadrant 1. 
We can see how before to implement the high speed response system the voltage decreases quickly up to the moment in which the crowbar system is activated $(-15 \mathrm{~V})$. The current increment in the mosfet is not enough to damp the effect of the lack of current provided by the opposite power module.

When the high speed response system is implemented, the current increases quickly after detecting the fault. Thanks to this phenomenon the magnet voltage is clamped around the $0 \mathrm{~V}$ and is kept in a safe situation that avoids the use of the crowbar system.

\section{Fault in quadrant 2, receptor mode.}

The graphs below show the converter behavior when one of the two power modules fails in the second quadrant in two different situations: Before to implement the high speed response system and after the implementation. Previously to cause the power module fault the working point is $-6 \mathrm{~V}$ and $300 \mathrm{~A}$ $(150 \mathrm{~A} \times 2)$.
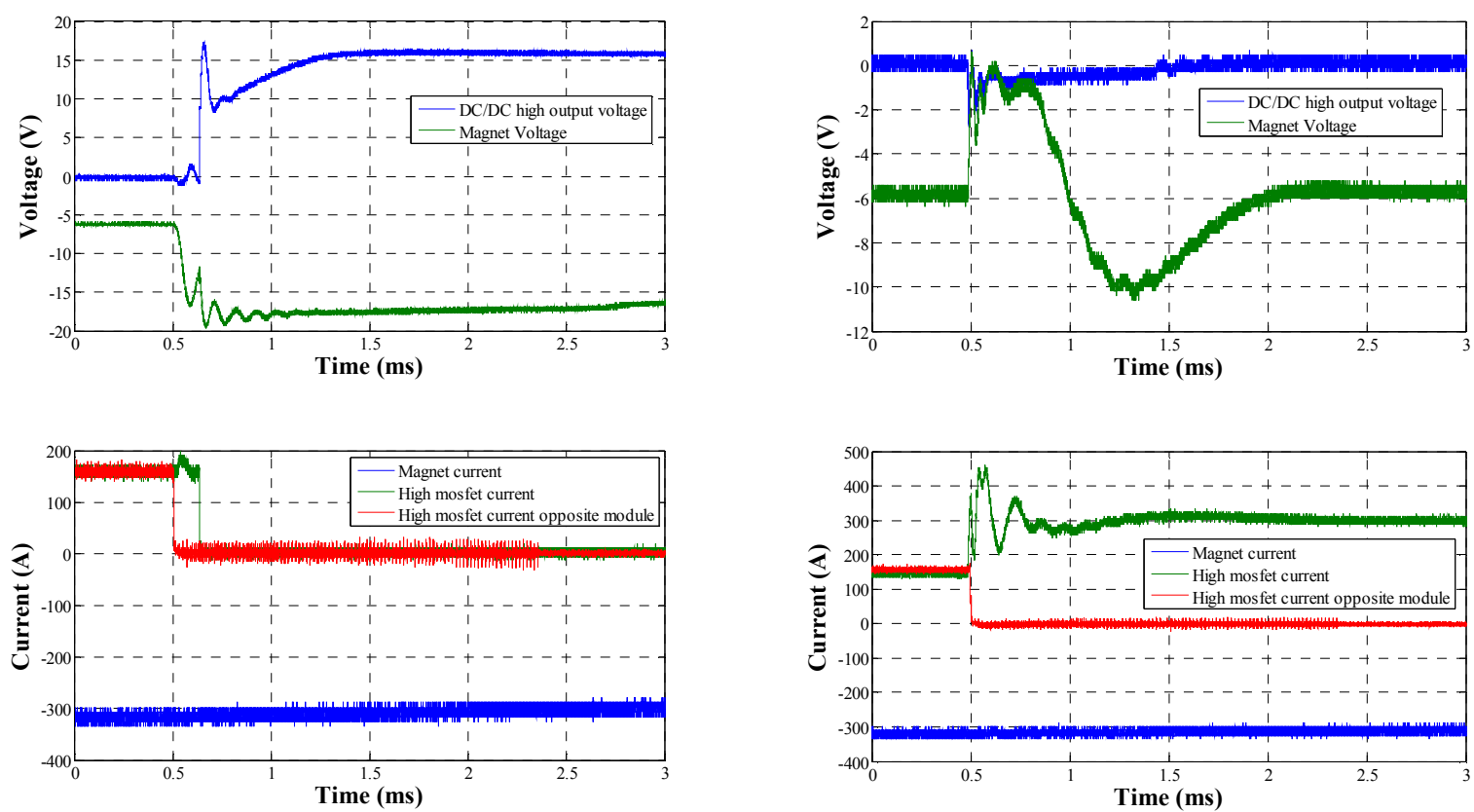

Fig. 14: Comparative of power module fault before (left column) and after (right column) implementing the high speed response system in quadrant 2.

We can see how before to implement the high speed response system the voltage decreases quickly up to the moment in which the crowbar system is activated $(-15 \mathrm{~V})$. The increment of current in the mosfet is not enough to damp the effect of the lack of current provided by the opposite power module.

When the high speed response system is implemented, the mosfet current increases quickly after detecting the fault. Thanks to this phenomenon the magnet voltage is clamped around the $0 \mathrm{~V}$ and the magnet is kept in a safe situation that avoids the use of the crowbar system.

The scope captures show how the system response to big perturbations is not ideal, a sub-damped behavior is presented.

\section{Conclusions.}

A new topology and control strategy are developed to comply with nominal LHC requirements. These modifications have been successfully implemented in a new converter prototype.

In next future, HL-LHC upgrade will demand new 4-quadrant converters, with a higher level of operating currents. It will be possible to feed these magnetic circuits with several power modules in parallel. A redesign could be done considering an undetermined number of power modules in parallel. 


\section{References}

[1] Y. Thurel, D. Nisbet, B. Favre, Four Quadrant 120A, 10V Power Converters for LHC, CERN, Geneva, Switzerland.

[2] C. de Almeida-Martins, Modélisation et Commande de l'Etage Linéaire d'un Convertisseur 4-Q à Mosfet, CERN, Geneva, Switzerland.

[3] F. Bordry, A. Dupaquier, High Current Low Voltage Power Converters for LHC Present Development Directions, E.P.A.C. 1996, Stiges, Spain.

[4] P. Cussac, F. Bordry, A. Dupaquier, High Precision and High Frequency Four-Quadrant Power Converter, E.P.E. 1999, Lausanne, Switzerland.

[5] G. Kniegl, R. Weber, F. Bordry, A. Dupaquier, Four-Quadrant Converter [ $\pm 600 \mathrm{~A}, \pm 12$ V] Prototype for LHC, P.A.C. 99, New York, USA. 